\title{
Correlação entre a Cobertura de Nuvens e Raios Gama ao Nível do Solo em São José dos Campos, SP.
}

Mauro Angelo Alves, Inácio Malmonge Martin, Instituto Tecnológico de Aeronáutica, SP.

\section{Copyright 2014, SBGt - Sociedade Brasileira de Geofísica}

Este texto foi preparado para a apresentação no VI Simpósio Brasileiro de Geofísica, Porto Alegre, 14 a 16 de outubro de 2014. Seu conteúdo foi revisado pelo Comitê Técnico do VI SimBGf, mas não necessariamente representa a opinião da SBGf ou de seus associados. É proibida a reprodução total ou parcial deste material para propósitos comerciais sem prévia autorização da SBGf.

\section{Resumo}

Medidas simultâneas efetuadas com intervalo de amostragem de um minuto da cobertura de nuvens em três níveis de altura $(250-2000 \mathrm{~m}, 2000-6000 \mathrm{~m}$ e $6000-8000 \mathrm{~m}$ ) e contagens de radiação gama obtidas ao nível do solo foram realizadas em maio e junho de 2014. Resultados mostram existir um grau de correlação entre a cobertura de nuvens e a contagens de radiação. Essa correlação pode ser causada pela variação da quantidade local de gás radônio-222, ou então pela produção de radiação devido à aceleração de elétrons originário de raios cósmicos por campos elétricos no interior de nuvens.

\section{Introdução}

O ambiente está exposto à radiação ionizante produzida por várias fontes existentes na natureza. As principais fontes de radiação natural são os raios cósmicos e o decaimento de isótopos radioativos presentes na crosta terrestre (urânio-238, tório-232, potássio-40) e no ar (radônio-222). Muitas vezes referida como radiação de fundo natural, a radiação a partir destas fontes naturais varia ao redor do mundo de acordo com fatores como altitude, latitude, geologia local, fenômenos meteorológicos e eventos geofísicos. A influência de raios cósmicos na radiação ambiental é já bem conhecida. Estudos sobre o assunto vêm sendo conduzidos desde o início do Século $X X$, sendo que Victor Hess ganhou o Prêmio Nobel em Física em 1936 pela descoberta de raios cósmicos. McCarthy \& Parks $(1984,1985)$ registraram a produção de raios $X$ associada a tempestades tropicais com detectores instalados em balões estratosféricos e aeronaves. Fishman et al. (1994) descreveram pela primeira vez a produção de raios gama por descargas elétricas na alta atmosfera. Moore et al. (2001) usaram cintiladores de $\mathrm{Nal}(\mathrm{TI})$ localizados em alta montanhas para detectar flashes de raio gama associados á ocorrência de relâmpagos. Gurevich et al. (1992) sugerem que fótons energéticos são produzido a partir da colisão de elétrons relativísticos com átomos no ar e da liberação de energia sob a forma de radiação (bremsstrahlung). Além de raios $X$ e gama, existem sólidas evidências que nêutrons também são produzidos por descargas elétricas. Shah et al. (1985) descreveram pela primeira vez a observação da produção de nêutrons por descargas elétricas atmosféricas. Este tipo de fenômeno tem sido, por exemplo, observado em observatórios localizados ao nível do mar ou a baixa altitude (Kuzhevsky, 2004; Martin \& Alves, 2010) e no topo de montanhas (Chubenko et al., 2008). Variações no fluxo de nêutrons térmicos também podem estar relacionadas a fenômenos sísmicos; Alekseenko et al. (2010) descrevem a existência de variações sazonais no fluxo de nêutrons terrestres causadas por efeitos de maré na crosta terrestre. Salikhov et al. (2013) descrevem o aumento no fluxo de nêutrons e raios gama no ambiente antes da ocorrência de terremotos. A radiação ionizante proveniente do espaço sideral também pode interferir em processos meteorológicos e atmosféricos. Gurevich et al. (1992) sugeriram que relâmpagos e outros tipos descargas elétricas atmosféricas podem ser iniciados pela colisão energética de raios cósmicos com átomos da atmosfera. Em um trabalho pioneiro, Svensmark e Friis-Christensen (1997) sugerem que existe uma forte correlação entre a modulação da incidência de raios cósmicos pela variação do campo magnético solar e a cobertura de nuvens no planeta. Recentemente, Svensmark et al. (2013) realizaram um estudo no qual foi mostrado em condições de laboratório que partículas produzidas pela ionização por raios gama na atmosfera formam núcleos de condensação, sugerindo que raios cósmicos podem influenciar a formação de nuvens. Em território nacional, Alves \& Martin, 2011; e Martin et al., 2013 vêm realizando estudos sobre a variação da radiação gama ambiental e sua correlação com parâmetros atmosféricos.

Baseado nesses estudos, procuramos determinar se existe uma correlação entre a radiação gama natural próxima ao nível do solo e a cobertura de nuvens para uma localidade.

\section{Metodologia}

Contagens de radiação gama e $X$ no intervalo de energia de $30 \mathrm{keV}$ a $10 \mathrm{Mev}$ são coletadas por um cintilador de $\mathrm{Nal}(\mathrm{TI})$ acoplado a uma fotomultiplicadora, sendo que o cristal e a fotomultiplicadora estão alojados em uma carcaça de alumínio para proteção mecânica (Modelo 44-20 Ludlum, USA). A fotomultiplicadora é alimentada por uma fonte alta tensão de $1400 \mathrm{~V}$, gerada a partir de uma tensão de entrada de 12 VDC. Os sinais de pulsos gerados pela radiação no cristal são amplificados e digitalizados por um sistema compacto (AWARE Electronics EUA). Um software específico detecta os sinais digitalizados e os grava em computador PC A cobertura de nuvens é medida com um radiômetro infravermelho operando entre $9-14 \mu \mathrm{m}$ (modelo CIR$4 \mathrm{~V}$, ATMOS Sarl, França). Este instrumento mede a fração do céu coberta por nuvens $(0 \%-100 \%)$ entre 250 a 8000 m em três níveis: 250 a 2000 m, 2000 a $6000 \mathrm{~m}$, e 6000 a $8000 \mathrm{~m}$. Ambos os instrumentos estão instalados em São José dos Campos, SP (23 12' $45^{\prime \prime} \mathrm{S}, 45^{\circ} 52^{\prime} 20^{\prime \prime} \mathrm{W}$, alt. $620 \mathrm{~m}$ ). O radiômetro infravermelho se encontra no alto da Torre de Observação de Fenômenos Atmosféricos do Instituto de Aeronáutica e Espaço, Divisão de Ciências Atmosféricas (IAE-ACA), com altura aproximada de 
25 m. O detector de raios gama está abrigado em um container equipado com ar condicionado próximo à base da Torre de Observação de Fenômenos Atmosféricos (Fig.1). A Fig. 2 mostra o radiômetro infravermelho no alto da torre, e a Fig. 3 mostra o detector de raios gama no interior do container climatizado.

O detector de raios gama e o radiômetro infravermelho coletam initerruptamente dados a intervalos de 1 minuto.

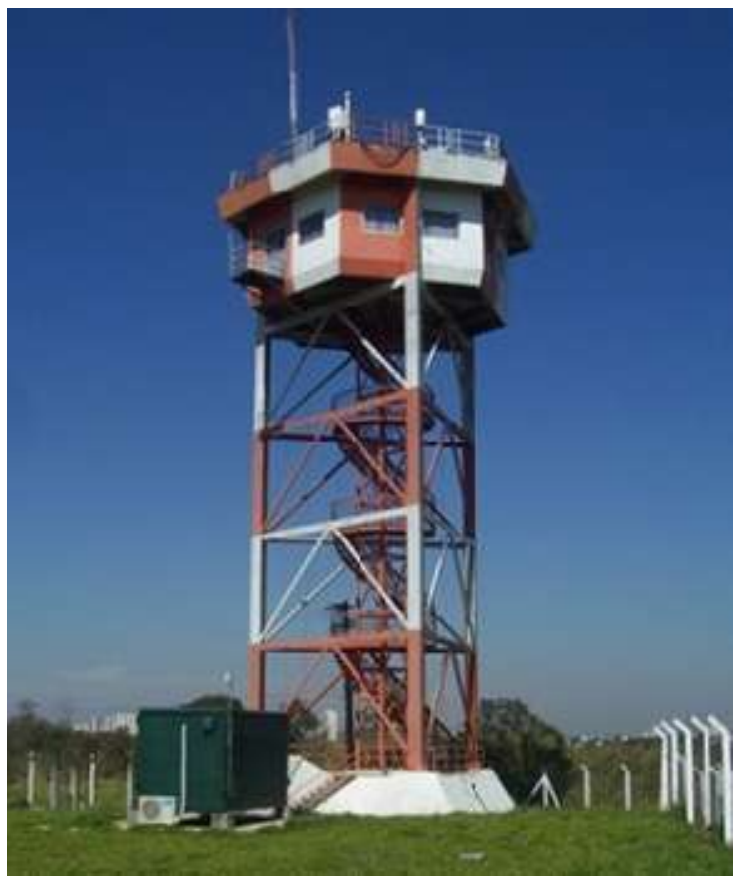

Figura 1 - Torre de Observação de Fenômenos Atmosféricos (IAE-ACA).

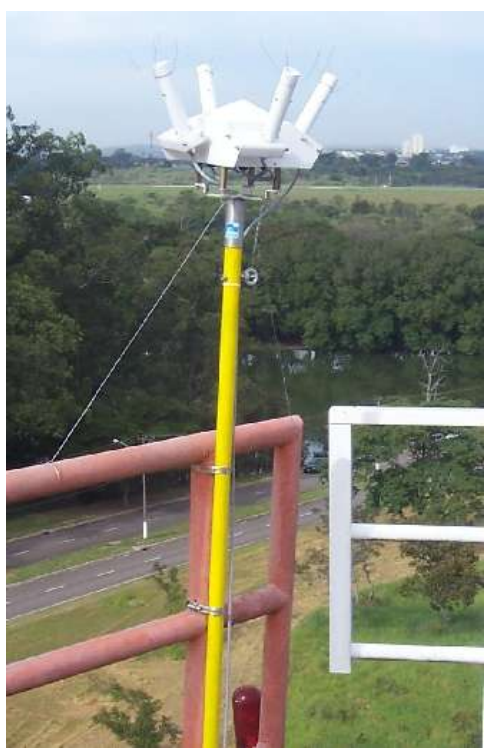

Figura 2 - Radiômetro infravermelho.

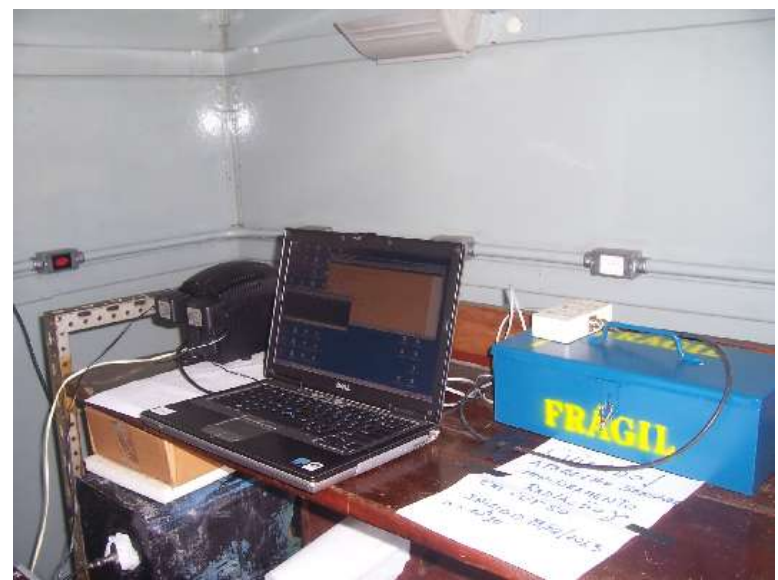

Figura 3 - Sistema compacto de detecção de raios gama. O cintilador encontra-se dentro da caixa de metal azul.

\section{Resultados}

Duas séries de tempo de nebulosidade e contagem de raios gama foram coletadas entre 06/05/2014 e 31/05/2014 (26 dias), e 01/06/2014 e 24/06/31 (2 dias). Os resultados são mostrados nas Figs. 4a e 4b. Nestas figuras, $\mathrm{N} 1$ corresponde à cobertura de nuvens no nível de 250 a 2000 m, N2 no nível de 2000 a 6000 m, e N3 no nível de 6000 a $8000 \mathrm{~m}$. O período de coleta de dados foi relativamente seco (poucas chuvas), o que é típico do fim da primavera e inicio do inverno na região. $\mathrm{Na}$ primeira série (Fig. 4a), observamos um evento (Evento I, dia 13, 19/05/2014) e que corresponde está associado a ocorrência de chuvas. Durante chuvas, é comum ocorrer o processo de radon washout que consiste na absorção por gotas de chuva do gás radônio-222 presente na coluna atmosférica e o seu transporte para o solo, ocasionado assim um aumento na contagem da radiação local. A cobertura de nuvens após esse dia é reduzida, o que corresponde à passagem da frente atmosférica associada às chuvas observadas no período.

Com relação a outras variações na contagem de raios gama, observamos que em geral existe uma maior correlação entre a cobertura de nuvens nos níveis $\mathrm{N} 1 \mathrm{e}$ $\mathrm{N} 2$ e as contagens de raios gama. Por exemplo, durante os intervalos I a IX (Fig. 4a e 4b) observamos existir certo grau de correlação entre aumentos nas contagens de radiação e variações na cobertura de nuvens. Uma inspeção mais cuidadosa destas figuras. parece indicar que os picos nas contagens da radiação gama estão associados a uma maior cobertura de nuvens, especialmente para os intervalos I, II, III, VII e VIII.

\section{Discussão e Conclusões}

Os resultados apresentados neste trabalho são preliminares e referem a um período no qual chuvas são menos comuns. Este estudo continua e será prolongado para incluir períodos com chuvas mais frequentes e intensas (verão, na região). 

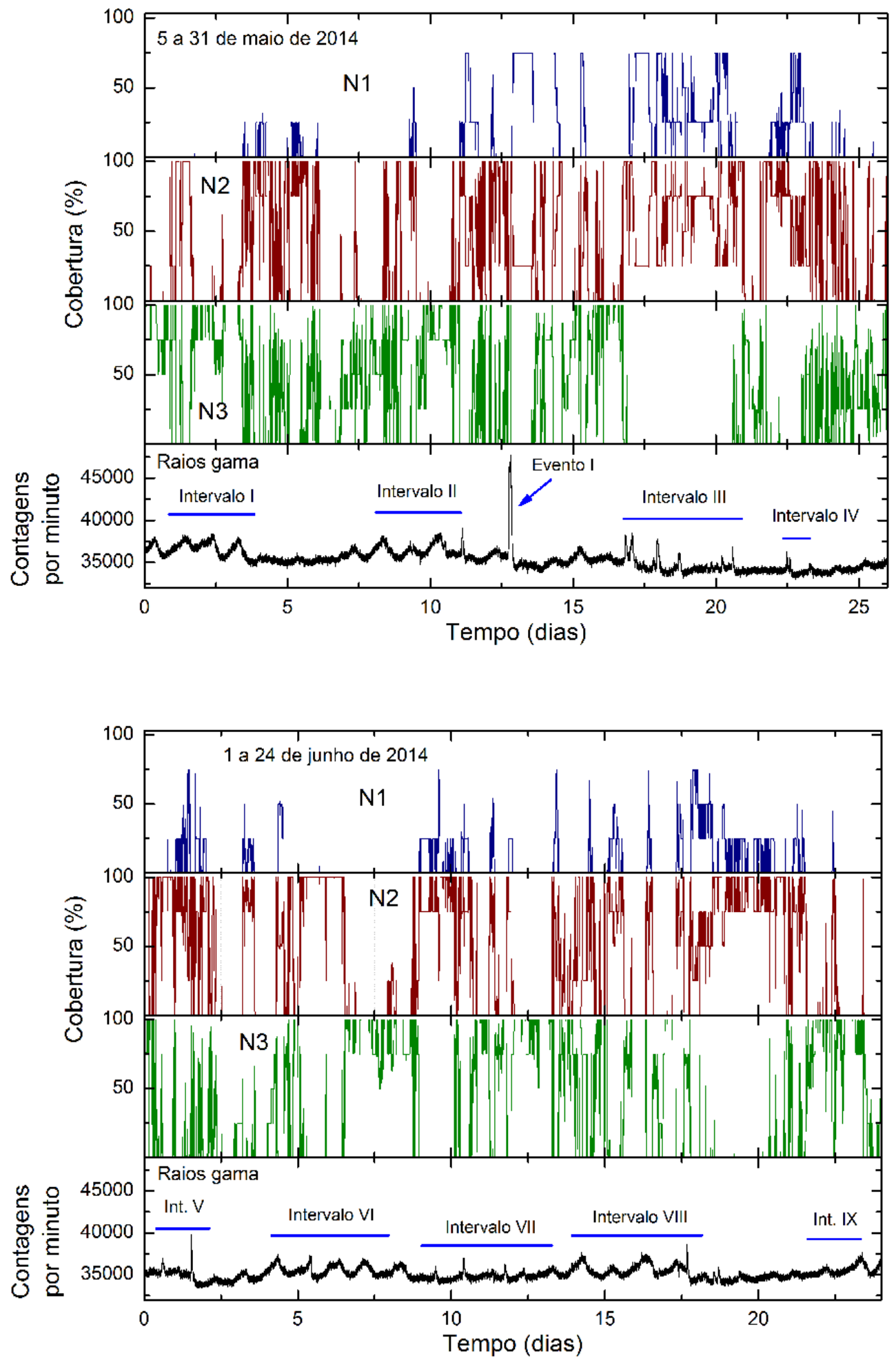

Figuras $4 a$ (acima) e $4 b$ (abaixo). Variação na cobertura de nuvens em três níveis de altura (N1, 250 a $2000 \mathrm{~m}$; N2, 2000 a $6000 \mathrm{~m}$; N3 6000 a 8000 m) e contagem de radiação gama. Os dados exibidos na Figura 4a foram coletados entre 6 a 31 de maio de 2014; e na Figura 4b, entre 1 a 24 de junho de 2014. 
No entanto, os resultados obtidos indicam que parece existir uma correlação entre a cobertura de nuvens em níveis inferiores a $6000 \mathrm{~m}$ e a variação na contagem de radiação gama ao nível do solo. Uma hipótese para explicar os resultados obtidos está relacionada ao fato de que a crosta terrestre libera continuamente para a atmosfera o gás radônio-222. Este gás se acumula na atmosfera próxima ao solo quando a turbulência atmosférica é reduzida. Com a aproximação de frentes frias, que produzem um aumento na cobertura de nuvens, a turbulência próxima ao solo aumenta, e o por processo de mistura, o gás radônio é distribuído de maneira mais homogênea na atmosfera. Outra hipótese para explicar o aumento observado nas contagens de radiação gama envolve a interação da radiação cósmica com nuvens mais densas (observadas em níveis mais baixos); elétrons gerados por raios cósmicos podem ser acelerados por campos elétricos intensos no interior de nuvens do tipo cumulonimbus (nuvens produtoras de chuva), dando origem a radiação bremsstrahlung (Vashenyuk et al. 2011).

\section{Agradecimentos}

Os autores agradecem à CAPES e ao CNPq pelo apoio financeiro, ao Instituto de Aeronáutica e Espaço, Divisão de Ciências Atmosféricas (IAE-ACA) e ao Departamento de Ciência e Tecnologia Aeroespacial (DCTA) pelo uso das instalações da Torre de Observações Meteorológicas, e ao Instituto Tecnológico de Aeronáutica (ITA) pelo apoio na realização deste trabalho.

\section{Referências}

Alekseenko VV, Gavriluk YM, Kuzminov VV, Stenkin YM, Tidal effect in the radon-due neutron flux from the Earth's crust Journal of Physics: Conference Series 203, 012045,1, 2010

Alves MA \& Martin IM. Monitoração de Radiação Gama Ambiental: Observação de Variações Diurnas e Efeitos Atmosféricos em São José dos Campos, SP. In: 63a Reunião Anual da SBPC, 2011, Goiânia. Anais da 63a Reunião Anual da SBPC, 2011.

Chubenko AP, Shepetov AL, Antonova VP, Chubenko PA, Kryuko SV, The influence of background radiation on the events registered in a neutron monitor at mountain heights, J. Phys. G, Nucl. Part. Phys 35, 085202, 2008.

Fishman, GJ, Bhat, PN, Mallozzi R, Horack JM, Koshut T, Kouveliotou, C, Pendleton, GN, Meegan CA, Wilson RB, Paciesas WS, Goodman SJ, Christian HJ, Discovery of Intense Gamma-Ray Flashes of Atmospheric Origin. Science 264 (5163): 1313-1316 1994

Gurevich AV, Milikh GM, Roussel-Dupré R. Runaway electron mechanism of air breakdown and preconditioning during a thunderstorm. Physics Letters A 165 (5-6): 463, 1992.
Kuzhevsky BM, Neutrons Born In Lightning, Moscow Univ. Phys. Bull. 5, 14, 2014.

Martin IM, Alves MA, Observation of a possible neutron burst associated with a lightning discharge? Journal of Geophysical Research-Space Physics, 115 (2010), A00E11

Martin I M, Alves MA, Ferro MA, Gomes MP, Pinto MLA, Antonio FL. Measurements of $X-$ and gamma radiation at ground level and their correlation with atmospheric electric discharges and rainfall in São José dos Campos, SP, Brazil. Thirteenth International Congress of the Brazilian Geophysical Society, Rio de Janeiro, 2013

McCarthy M \& Parks, GK. X-rays from thunderstorm and lightning active regions, Proceedings of 7 th International Conference of Atmospheric Electricity, 508,1984

McCarthy M. \& Parks GK. Further observations of Xrays inside thunderstorms, Geophysical Research Letters, Vol. 12, No. 6, p. 393, 1985.

Moore, CB, Eack KB, Aulich GD, Rison W (2001), Energetic radiation associated with lightning steppedleaders. Geophys. Res. Lett. 28, 11, 2141.

Salikhov NM, et al., Observation of the prior earthquake effect on the flux of environmental neutrons, gammaradiation, and on the local electric field in Tien Shan mountain. Artigo não publicado (no prelo).

Shah GN, Razdan H, Bhat CL, Ali QM. Neutron generation in lightning bolts, Nature $313,773-775$, 1985.

Svensmark H \& Friis-Christensen E. Variation of cosmic ray flux and global cloud coverage - a missing link in solar-climate relationships, Jn. of Atmospheric and Solar-Terrestrial Physics, 59, 11, 1225-1232, 1997.

Svensmark H, Martin B E, Pedersen J. Response of cloud condensation nuclei (>50 nm) to changes in ionnucleation, Physics Letters A 377 2343-2347, 2013.

Vashenyuk EV, Balabin YV, Germanenko AV, Gvozdevsky BB. Study of radiation related with atmospheric precipitations. 32nd International Cosmic Ray Conference, Beijing 2011 